\title{
Quantum measurement of a solid-state qubit: A unified quantum master equation approach
}

\author{
Xin-Qi Li ${ }^{1,3}$ Wen-Kai Zhang, ${ }^{2}$ Ping Cui, ${ }^{3}$ Jiushu Shao, ${ }^{2}$ Zhongshui Ma, ${ }^{4}$ and YiJing Yan $^{3}$ \\ ${ }^{1}$ National Laboratory for Superlattices and Microstructures, Institute of Semiconductors, \\ Chinese Academy of Sciences, P.O. Box 912, Beijing 100083, China \\ ${ }^{2}$ State Key Laboratory of Molecular Reaction Dynamics, Institute of Chemistry, Chinese Academy of Sciences, Beijing 100080, China \\ ${ }^{3}$ Department of Chemistry, Hong Kong University of Science and Technology, Kowloon, Hong Kong \\ ${ }^{4}$ State Key Laboratory for Mesoscopic Physics and Department of Physics, Peking University, Beijing 100871, China
}

(Received 9 June 2003; revised manuscript received 24 September 2003; published 20 February 2004)

\begin{abstract}
Quantum measurement of a solid-state qubit by a mesoscopic detector is of fundamental interest in quantum physics and an essential issue in quantum computing. In this work, by employing a unified quantum master equation approach constructed in our recent publications, we study the measurement-induced relaxation and dephasing of the coupled-quantum-dot states measured by a quantum-point contact. Our treatment pays particular attention on the detailed-balance relation, which is a consequence of properly accounting for the energy exchange between the qubit and detector during the measurement process. As a result, our theory is applicable to measurement at arbitrary voltage and temperature. Both numerical and analytical results for the qubit relaxation and dephasing are carried out, and important features are highlighted in concern with their possible relevance to future experiments.
\end{abstract}

DOI: 10.1103/PhysRevB.69.085315

PACS number(s): 72.10.-d, 03.65.-w, 03.65.Yz

\section{INTRODUCTION}

Measuring a two-state quantum system (qubit) typically represents the long-standing and still controversial issue in quantum measurement. Ideally, based on the standard Copenhagen postulate, quantum measurement is described as a wave-function collapse, i.e., projects the qubit state to one of the possible eigenstates of the observed quantity with state-dependent probabilities. However, in practice, any realistic measurement is performed by a realistic device that itself is a physical system. Response of the measured system to the measuring device is in general a nontrivial problem, which has attracted considerable attention in recent years. ${ }^{1-18}$ This renewed interest also stems from the rapidly developing field of quantum computing, since the quantum-measurement procedure is needed, for instance, at the end of computation to read out the final results, or even in the course of computation for the purpose of error correction.

A possible implementation of the two-state quantum measurement is to consider a charge qubit being measured by a charge sensible detector, such that the transport current in the detector carries information of the measured qubit. The charge qubit can be either an extra electron stored in coupled quantum dots (CQD's), ${ }^{19}$ or an extra Cooper pair in superconducting box, ${ }^{7,13,17}$ meanwhile the detector can be a quantum-point contact $^{1-6,12,16}$ (QPC), or a single-electron transistor $^{7-11,17}$ (SET). In these studies, in addition to theoretical discussions, ${ }^{1-10,16,17}$ experimental results have also been reported. ${ }^{11-13}$

To study the effects of measurement on a quantum system, the standard procedure is to trace out the microscopic degrees of freedom of the detector, which would result in a reduced description in terms of quantum master equation (QME) for the relaxation and dephasing of the measured system. In the seminal work by Gurvitz, ${ }^{1}$ the quantum measurement of the charge state in coupled CQD's by a QPC was studied based on a reducing procedure from the many- particle wave function of the entire qubit-plus-detector system. This approach was also applied to study the breakdown of the Anderson localization in the presence of quantum measurement, ${ }^{14}$ and its conditional version was exploited to analyze the readout of the detector. ${ }^{15,18} \mathrm{By}$ an alternative means, Goan et al. derived a Lindblad QME for the same measurement setup, based on which a quantum trajectory description was developed for the single continuous measurement. ${ }^{5}$ The Lindblad QME obtained by Goan et al. has also been demonstrated to be equivalent to the Bloch equations derived by Gurvitz. ${ }^{5}$ However, we notice that their master equations would inevitably lead to certain peculiar features such as the always equal occupation probabilities on individual dots (sites) in the asymmetric qubit (disorder chain) after the completion of dephasing and relaxation. ${ }^{1,14}$

In this work, we revisit this well-defined quantummeasurement problem by employing a unified Markovian QME approach. ${ }^{20-22}$ We pay particular attention on the detailed-balance relation, which properly accounts for the energy exchange between the qubit and detector during the measurement process. Consequently, our approach is valid at arbitrary measurement voltage and temperature. It will show that the results in Refs. 1 and 5 break down at small voltage and the peculiar features in Refs. 1 and 14 survive only in high-voltage regime. Note that the measurement voltage can in a certain sense be interpreted as an effective temperature; ${ }^{15}$ thus the equal occupation probabilities on individual states may be viewed as the result of an effectively strong thermalization. To our knowledge, this kind of clarification lacks so far in literature. Recently, similar quantum measurement under arbitrary voltage is analyzed in terms of the noise spectrum of the detector output signal. ${ }^{10,16,17}$ In the large voltage regime, the noise spectrum is symmetric; and in small voltage regime, the noise spectrum becomes asymmetric. This change of spectral shape indicates a transition from classical to quantum. In the quantum regime, it is right the energy exchange between the qubit and detector that leads to 

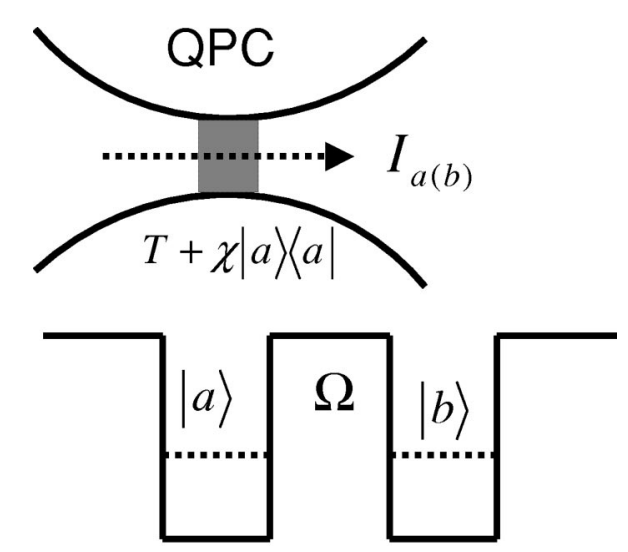

FIG. 1. Schematic diagram of a solid-state qubit (coupled quantum dots) being measured by a quantum-point contact (QPC). Occupation of the extra electron in different dots would have distinct influence on the transport current through the QPC, which makes it possible to draw out the qubit-state information. On the other hand, as a result of back action of the detector, quantum coherence of the qubit state would be destroyed.

the asymmetry of noise spectrum. Our present work, which deals with the measurement-induced qubit dephasing and relaxation under arbitrary voltage and temperature, thus provides an alternative perspective to elucidate the nature of energy exchange and its importance in describing quantum measurement.

The remainder of this paper is organized as follows. In the context of the considered quantum-measurement model, we outline in Sec. II the derivation of the QME for the reduced dynamics of the qubit. The detailed-balance property of our QME and its relation to the energy exchange processes accompanying the quantum measurement will be elaborated in the Appendix. In Sec. III, numerical results of the relaxation and dephasing behaviors of the measured qubit are presented, and discussions are highlighted to some important features resulting from the detailed balance. Section IV contributes to the relaxation and dephasing rates where the derived analytical formulas clearly describe effects of various measurement parameters, such as the bias voltage across the QPC and the temperature in the electronic reservoirs. Finally, in Sec. V we summarize the main results and implications of this work.

\section{QME APPROACH FOR QUANTUM MEASUREMENT: FORMAL RESULT}

\section{A. Model description}

Following the previous work, ${ }^{1,4,5}$ we consider here a coupled quantum dot (i.e., a solid-state qubit) measured by a quantum-point contact, as schematically shown in Fig. 1. To present a microscopic description for the measurement, assume the Hamiltonian of the entire qubit-plus-reserviros system as

$$
H=H_{\mathrm{qu}}+H_{\mathrm{res}}+H^{\prime}
$$

with

$$
\begin{gathered}
H_{\mathrm{qu}}=\epsilon_{a}|a\rangle\left\langle a\left|+\epsilon_{b}\right| b\right\rangle\langle b|+\Omega(|b\rangle\langle a|+| a\rangle\langle b|), \\
H_{\mathrm{res}}=\sum_{k} \epsilon_{k}^{\mathrm{L}} c_{k}^{\dagger} c_{k}+\sum_{q} \epsilon_{q}^{\mathrm{R}} d_{q}^{\dagger} d_{q}, \\
H^{\prime}=\sum_{k, q}\left(T_{q k}+\chi_{q k}|a\rangle\langle a|\right) c_{k}^{\dagger} d_{q}+\text { H.c. }
\end{gathered}
$$

The two terms in $H_{\text {res }}$ are for electrons in the two reservoirs (electrodes) labeled by " $L$ " and " $R$," respectively. The interaction Hamiltonian $H^{\prime}$ here describes the electron tunneling through QPC, e.g., from state $|q\rangle$ in the $R$ reservoir to state $|k\rangle$ in the $L$ reservoir, with the tunneling coupling amplitude of $\left(T_{q k}+\chi_{q k}|a\rangle\langle a|\right)$ that depends explicitly on the qubit-state. It is right this qubit-state dependence of the tunneling amplitude that makes it possible to draw out the qubitstate information from the transport current through QPC. In the above microscopic Hamiltonian, the detector is described in terms of second quantization to address the many-particle nature, meanwhile for the measured system (qubit) singleparticle description is adopted since there is only one extra electron in it. Here we denote the qubit states by $|a\rangle$ and $|b\rangle$, corresponding to the electron locating in the left and right dots. In this work we shall also introduce the qubit eigenstates $|1\rangle$ and $|2\rangle$, which are the superpositions of the dot states $|a\rangle$ and $|b\rangle$.

\section{B. Reduced description for the measured qubit}

Quantum measurement can be characterized by dephasing and relaxation of the measured system. In this section, we present a unified QME description for the reduced dynamics of the qubit which is subjected to the measurement of a QPC. Details of the formal derivation and the adopted approximations are referred to in Ref. 21 . Here we only outline the key procedure and the main results with respect to the measurement model in concern.

It is well known that in weak-coupling regime one can derive the QME by carrying out a second-order cumulant expansion with respect to the system-environment interaction Hamiltonian. In our case, we treat the qubit-statedependent tunneling Hamiltonian $H^{\prime}$ as perturbation, since it fully contains the coupling information between the qubit and detector. In the interaction picture with respect to the QPC reservoir Hamiltonian $H_{\text {res }}$ of Eq. (1c), the interaction Hamiltonian $H^{\prime}$ of Eq. (1d) becomes time dependent and reads (setting $\Delta_{k q} \equiv \epsilon_{k}^{\mathrm{L}}-\epsilon_{q}^{\mathrm{R}}$ )

$$
H^{\prime}(t)=\sum_{q, k}\left(T_{q k}+\chi_{q k}|a\rangle\langle a|\right) c_{k}^{\dagger} d_{q} e^{i \Delta_{k q} t}+\text { H.c. }
$$

Making connection with the formalism developed in Ref. 21, we denote $W_{q k}=T_{q k}+\chi_{q k}|a\rangle\langle a|$ and $f_{q k}^{\dagger}(t)=c_{k}^{\dagger} d_{q} e^{i \Delta_{k q} t}$, which are operators in the qubit and the stochastic bath reservoir subspaces, respectively, and recast Eq. (2) as $H^{\prime}(t)$ $=\Sigma_{q, k}\left[W_{q k} f_{q k}^{\dagger}(t)+W_{q k}^{\dagger} f_{q k}(t)\right]$. With this form and starting from the Liouville equation, the QME satisfied by the reduced density matrix can be derived after tracing out the 
microscopic degree of freedom of the QPC reservoirs, precisely following the procedures in Ref. 21 .

For simplicity, we assume $T_{q k} \equiv T$ and $\chi_{q k} \equiv \chi$, i.e., the tunneling amplitudes are reservoir-state independent. Accordingly, the interaction Hamiltonian simplifies to $H^{\prime}(t)$ $=W f^{\dagger}(t)+W^{\dagger} f(t)$, where

$$
W=T+\chi|a\rangle\langle a|, f^{\dagger}(t)=\sum_{k, q} c_{k}^{\dagger} d_{q} e^{i \Delta_{k q} t} .
$$

The measurement current fluctuation-induced dephasing and relaxation effects on the qubit are characterized by the interaction bath correlation functions, which in relation to the QPC detector shown in Fig. 1 can be carried out explicitly as

$$
\begin{gathered}
\widetilde{C}^{(+)}(t) \equiv\left\langle f^{\dagger}(t) f(0)\right\rangle=\sum_{k, q} e^{i \Delta_{k q} t} N_{\mathrm{L} k}\left(1-N_{\mathrm{R} q}\right), \\
\widetilde{C}^{(-)}(t) \equiv\left\langle f(t) f^{\dagger}(0)\right\rangle=\sum_{k, q} e^{-i \Delta_{k q} t}\left(1-N_{\mathrm{L} k}\right) N_{\mathrm{R} q} .
\end{gathered}
$$

Here, $\langle\cdots\rangle$ stands for the statistical average over both the left and right electron reservoirs, which are assumed to be in the local thermal equilibrium, with the Fermi-Dirac functions being given by $N_{\mathrm{L} k}=\left[e^{\beta\left(\epsilon_{k}^{\mathrm{L}}-\mu_{\mathrm{L}}\right)}+1\right]^{-1}$ and $N_{\mathrm{R} q}$ $=\left[e^{\beta\left(\epsilon_{q}^{\mathrm{R}}-\mu_{\mathrm{R}}\right)}+1\right]^{-1}$, respectively. Here, $\beta=1 /\left(k_{B} T\right)$ is the inverse temperature, and $\mu_{\mathrm{L}}$ and $\mu_{\mathrm{R}}$ are the chemical potentials that relate to the applied voltage across the detector by $\mu_{\mathrm{L}}-\mu_{\mathrm{R}}=e V$. The interaction bath spectrum is then defined as the Fourier transform of the reservoir-electron correlation function, ${ }^{21}$

$$
C^{( \pm)}( \pm \omega)=\int_{-\infty}^{\infty} d t \widetilde{C}^{( \pm)}(t) e^{ \pm i \omega t}
$$

It satisfies the detailed-balance relation of $C^{(+)}(\omega) /$ $C^{(-)}(-\omega)=e^{\beta(\omega+e V)}$.

With the above clarifications, the unified QME developed in Ref. 21 applied here to describe the dephasing and relaxation of the measured qubit can now be completely identified:

$$
\dot{\rho}=-i \mathcal{L} \rho-\mathcal{R} \rho,
$$

with $\mathcal{L}(\cdots) \equiv\left[H_{\mathrm{qu}},(\cdots)\right]$ being the qubit Liouvillian and $\mathcal{R}$ being the dissipation superoperator defined via the following compact form, ${ }^{21}$

$$
\mathcal{R} \rho=\frac{1}{2}\left[W^{\dagger}, \widetilde{W}^{(-)} \rho-\rho \widetilde{W}^{(+)}\right]+\text {H.c. }
$$

Formally, $\widetilde{W}^{( \pm)}$is related to the coupling operator $W$ as

$$
\widetilde{W}^{( \pm)}=C^{( \pm)}( \pm \mathcal{L}) W .
$$

Here, $C^{( \pm)}( \pm \mathcal{L})$ is a superoperator, specified by the qubit Liouvillian $\mathcal{L}$ and the interaction bath spectrum $C^{( \pm)}( \pm \omega)$. To obtain the explicit expressions of $C^{( \pm)}( \pm \mathcal{L})$, we further adopt the continuum and wideband approximations for the QPC reservoir electrons. Accordingly, the discrete summations in Eq. (4) can be replaced by the continuous integra- tions, $\quad \Sigma_{k} \Sigma_{q} \rightarrow g_{\mathrm{L}} g_{\mathrm{R}} \iint d \epsilon_{k}^{\mathrm{L}} d \epsilon_{q}^{\mathrm{R}}$, where the energyindependent density of states (DOS) $g_{\mathrm{L}}$ and $g_{\mathrm{R}}$ are introduced for the two reservoirs. The analytical expressions for $C^{( \pm)}( \pm \mathcal{L})$ can then be readily integrated out as

$$
C^{( \pm)}( \pm \mathcal{L})=2 \pi g_{\mathrm{L}} g_{\mathrm{R}}\left[\frac{x}{1-e^{-\beta x}}\right]_{x= \pm(\mathcal{L}+e V)} .
$$

Equations (6)-(9) constitute the QME formulation that contains the full effects of measurement on the qubit and will serve as the starting point of the following studies.

\section{Comments and discussions}

Let us start with the high measurement voltage limit $(e V \gg \mathcal{L})$, in which the applied measurement voltage is much larger than the internal energy scale of the qubit. In this case, the superoperator $C^{( \pm)}( \pm \mathcal{L})$ of Eq. (9) reduces to a $c$ number,

$$
C^{( \pm)}( \pm \mathcal{L}) \rightarrow C^{( \pm)}(0)= \pm 2 \pi g_{\mathrm{L}} g_{\mathrm{R}} \frac{e V}{1-e^{\mp \beta e V}} .
$$

With this approximation, Eq. (7) recovers the QME derived in Ref. 5, i.e.,

$$
\mathcal{R} \rho \simeq-C^{(-)}(0) \mathcal{D}[W] \rho-C^{(+)}(0) \mathcal{D}\left[W^{\dagger}\right] \rho,
$$

with $\mathcal{D}[W] \rho=W \rho W^{\dagger}-\frac{1}{2}\left[W^{\dagger} W, \rho\right]_{+}$and $\mathcal{D}\left[W^{\dagger}\right] \rho$ being defined similarly by swapping between $W$ and $W^{\dagger}$. It is easy to show $^{5}$ that the QME of Eq. (11) is in fact also equivalent to the Bloch equation derived by Gurvitz. ${ }^{1}$

Our QME formulation in Eqs. (6)-(9) is valid for arbitrary measurement voltage. In contrast with the $c$ number of $C^{( \pm)}(0)$, the operator nature of $C^{( \pm)}( \pm \mathcal{L})$ in our QME formulation properly describes not only the dephasing but also the important energy exchange between the qubit and detector. Physically, the quantum-measurement-induced dephasing and relaxation on the qubit are originated from the current fluctuations in the detector. ${ }^{10,16,17}$ As mentioned earlier, the current fluctuations are characterized by the correlation functions $\widetilde{C}^{( \pm)}(t)$ of Eq. (4), and their spectra satisfy the detailed-balance relation of $C^{(+)}(\omega) / C^{(-)}(-\omega)=e^{\beta(\omega+e V)}$ at arbitrary temperature and measurement voltage [cf. Eq. (9)]. Consequently, $C^{( \pm)}( \pm \mathcal{L})$ manifests the back action of the detector on the qubit by correlating the measurement current fluctuations with the qubit dissipations, leading to our QME in Eqs. (6)-(9) satisfying the detailed-balance relation. More specifically, the energy exchange between the qubit and detector can be described as follows. $C^{(+)}(\mathcal{L})$ accounts for the current fluctuations associated with electron tunneling in the detector from the left to the right reservoirs, accompanied by energy absorption from the qubit, while $C^{(-)}(-\mathcal{L})$ corresponds to tunneling from the right reservoir to the left one, accompanied by energy emission to the qubit. The energy exchange characterized by $C^{( \pm)}( \pm \mathcal{L})$, i.e., the currentfluctuation spectrum $C^{( \pm)}( \pm \omega)$ correlated with qubit Liouvillian $\omega=\mathcal{L}$, is an essential feature hold by our QME that manifests the important detailed-balance relation. This issue 

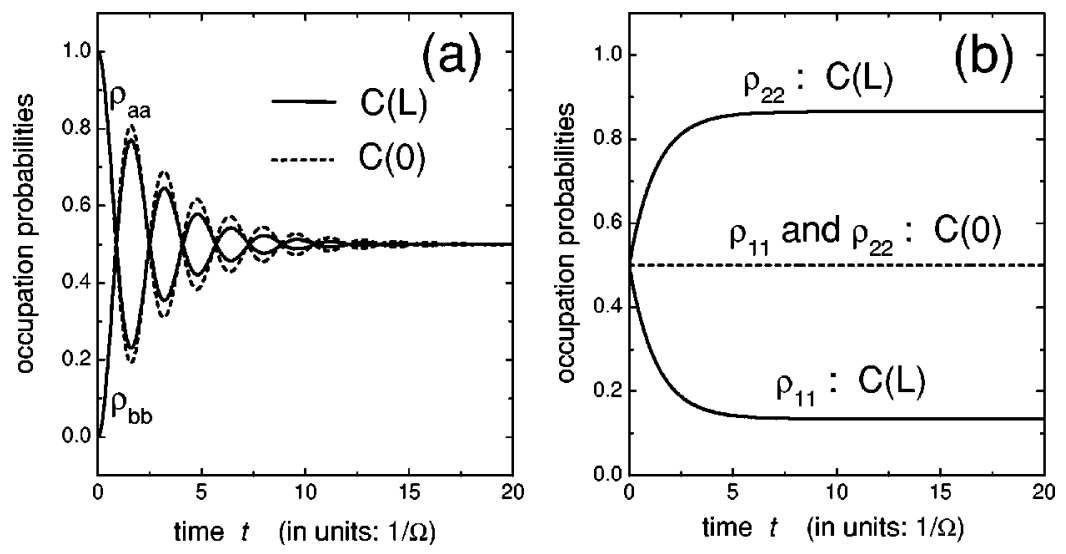

FIG. 2. Measurement-induced qubit relaxation in (a) the individual dot-state representation, and (b) the eigenstate representation. The results in the presence and the absence of the detailed balance are symbolized by " $C(\mathcal{L})$ " and “ $C(0)$, , respectively.

is further elaborated in the Appendix by carrying out the explicit expression of the QME in terms of the qubit atomic operators.

\section{MEASUREMENT-INDUCED DEPHASING AND RELAXATION}

We are now in the position to highlight the essential role and impact of the detailed balance on the qubit dephasing and relaxation under measurement. In order to have a close comparison with the previous results that neglect the detailed-balance effects, ${ }^{1,5}$ instead of the QME given explicitly in the Appendix in terms of the qubit atomic operators $\sigma_{z}$ and $\sigma^{ \pm}$, in this section we would like to elaborate it in the individual dot-state basis. For clarity, our results will be presented for the symmetric and asymmetric qubit cases, separately, in the following two sections.

\section{A. Symmetric case: $\epsilon_{a}=\epsilon_{b}$}

In the individual dot-state representation $\{|a\rangle,|b\rangle\}$, the coupling operator $W$ takes a matrix form

$$
W=\left[\begin{array}{cc}
T+\chi & 0 \\
0 & T
\end{array}\right],
$$

and its spectral conjugate in Eq. (8) is

$$
\widetilde{W}^{( \pm)}=\left[\begin{array}{cc}
\widetilde{W}_{a a}^{( \pm)} & \widetilde{W}_{a b}^{( \pm)} \\
\widetilde{W}_{b a}^{( \pm)} & \widetilde{W}_{b b}^{( \pm)}
\end{array}\right] .
$$

The involving $\widetilde{W}^{( \pm)}$-matrix elements can be evaluated readily via the standard operator algebra. To simplify the notation, let us denote

$$
\begin{aligned}
& \lambda_{ \pm} \equiv\left[C^{( \pm)}( \pm \Delta)+C^{( \pm)}(\mp \Delta)\right] / 4, \\
& \bar{\lambda}_{ \pm} \equiv\left[C^{( \pm)}( \pm \Delta)-C^{( \pm)}(\mp \Delta)\right] / 4 .
\end{aligned}
$$

Here $\Delta=E_{1}-E_{2}$, with $E_{1}$ and $E_{2}$ being the qubit eigenenergies.

For the symmetric qubit $\left(\epsilon_{a}=\epsilon_{b}=\epsilon_{0}\right)$, we have then

$$
\widetilde{W}_{a a}^{( \pm)}=(T+\chi / 2) C^{( \pm)}(0)+\chi \lambda_{ \pm},
$$

$$
\begin{gathered}
\widetilde{W}_{b b}^{( \pm)}=(T+\chi / 2) C^{( \pm)}(0)-\chi \lambda_{ \pm}, \\
\widetilde{W}_{b a}^{( \pm)}=-\widetilde{W}_{a b}^{( \pm)}=\chi \bar{\lambda}_{ \pm} .
\end{gathered}
$$

Substituting Eq. (15) into the formal QME [Eqs. (6) and (7)], the Bloch equations for the reduced density matrix in the dot-state representation can be readily obtained. For instance, the off-diagonal density-matrix element satisfies

$$
\begin{aligned}
\dot{\rho}_{a b}= & -i\left(\epsilon_{a}-\epsilon_{b}\right) \rho_{a b}+i \Omega\left(\rho_{a a}-\rho_{b b}\right)-\chi^{2}\left(\lambda_{+}+\lambda_{-}\right) \rho_{a b} \\
& -\frac{\chi^{2}}{2}\left(\bar{\lambda}_{+}-\bar{\lambda}_{-}\right)\left(\rho_{a a}+\rho_{b b}\right)
\end{aligned}
$$

For simplicity, we have assumed here the tunneling coefficients $T$ and $\chi$ to be real. It is easy to see that in the absence of detailed balance, i.e., $C^{( \pm)}( \pm \Delta) \rightarrow C^{( \pm)}(0)$, Eq. (16) reduces to

$$
\begin{aligned}
\dot{\rho}_{a b}= & -i\left(\epsilon_{a}-\epsilon_{b}\right) \rho_{a b}+i \Omega\left(\rho_{a a}-\rho_{b b}\right)-\frac{\chi^{2}}{2}\left[C^{(+)}(0)\right. \\
& \left.+C^{(-)}(0)\right] \rho_{a b},
\end{aligned}
$$

which is nothing but the result derived in Refs. 1 and 5.

Under the quantum measurement, a pure state of the qubit state evolves into a statistical mixture. Figure 2 shows such evolution by plotting the time-dependent occupation probabilities on the individual dot states. In the following numerical studies, the relevant parameters are adopted as follows: the applied voltage over the QPC $e V=\Omega$, the inverse temperature $\beta=1 / \Omega$, the DOS in both electron reservoirs $g_{\mathrm{L}}$ $=g_{\mathrm{R}}=2 / \Omega$, and the tunneling amplitudes $T=\Omega$ and $\chi$ $=0.15 \Omega$. In the dot representation as shown in Fig. 2(a), despite certain quantitative difference in short time scale, common final occupation probability of $1 / 2$ in each dot is approached, irrelevant to the detailed balance being satisfied or not. Physically, due to the measurement-induced dephasing, a transition occurs for the qubit electron tunneling from the coherent to incoherent regime. In the coherent regime, the tunneling results in the well-known Rabi oscillations. In the incoherent regime, no phase correlation exists between the tunneling events, and the readout appears as telegraphic signals. In the symmetric case, owing to $\epsilon_{a}=\epsilon_{b}$, the final equal occupation probability of $1 / 2$ in each dot is anticipated. 

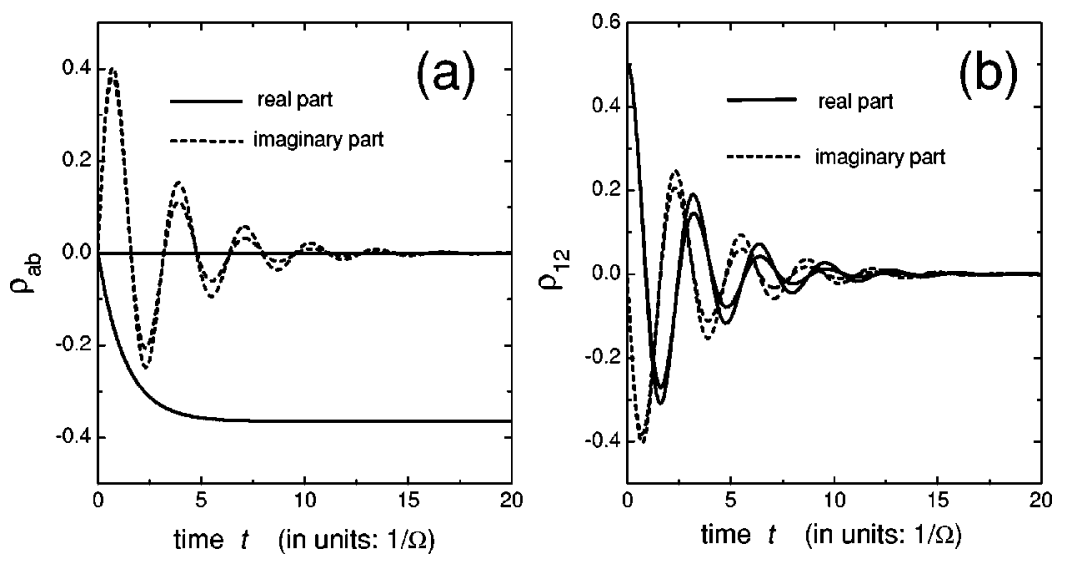

FIG. 3. Measurement-induced dephasing in (a) the individual dot-state representation, and (b) the eigenstate representation. The real part and imaginary parts of the off-diagonal matrix element are plotted by the solid and dashed curves, respectively. In (a), the result in the absence of detailed balance is shown by the constant zero solid line and the dashed curve with larger oscillation amplitude. The other two curves are for the detailed-balance preserved result. Similarly, in (b), the curves with smaller and larger amplitudes correspond, respectively, to the detailed-balance hold and unhold results.
However, as shown by Gurvitz, ${ }^{1}$ in the asymmetric case (i.e., for nonidentical coupled dots), final equal occupation probability of $1 / 2$ in each dot would also be approached. Similar confusing feature also existed in the breakdown of the Anderson localization, where equal occupation probabilities on each site of the disordered chain were found. ${ }^{14}$ This peculiar feature is only valid in the limit of large measurement voltage, which causes an effective thermalization in terms of an effective temperature. The equal stationary occupation in a general asymmetric case is however unphysical; it violates the detailed balance since it does not properly account for the energy exchange between the measured system and the detector.

To reveal the significant implication of the detailed balance, let us transform the result in Fig. 2(a) into the qubiteigenstate representation, as shown in Fig. 2(b). Viewing that initially the electron locates in the left dot, which is equivalent to $1 / 2$ probability in each eigenstate of the symmetric qubit, the constant dashed line in Fig. 2(b) indicates that equal occupation probabilities on the two eigenstates would be unaffected if the detailed balance could be neglected. However, the proper relaxation between the eigenstates will result in quite different occupation probabilities as shown by the solid curves in Fig. 2(b).

Despite the drastic consequence of the detailed balance on relaxation, our QME also stimulates an interesting issue in dephasing. In Fig. 3(a) the dephasing behavior is described by the off-diagonal density-matrix element in the dot-state representation. We see that in the absence of detailed balance, complete dephasing between the dot states takes place at the long measurement time limit. However, in the presence of detailed balance, the real part of $\rho_{a b}$ approaches a nonzero constant. We notice that a similar feature of nonzero offdiagonal matrix element in dot-state basis appears also in Ref. 18 [see Eq. (11) there] by coupling the qubit with an additional thermal bath. In contrast, our result stems merely from the coupling with the detector, owing to the fact that our theory properly accounts for the energy exchange between the qubit and detector, and thus its consequence on dephasing and relaxation. Mathematically, noting that $\rho_{a a}$ $=\rho_{b b}=0.5$ as $t \rightarrow \infty$, the off-diagonal matrix element approaches asymptotically to a nonzero value of $\rho_{a b}(t)$ $\rightarrow 0.5\left(\bar{\lambda}_{-}-\bar{\lambda}_{+}\right) /\left(\lambda_{-}+\lambda_{+}\right)$via Eq. (16), rather than $\rho_{a b}(t)$ $\rightarrow 0$ via Eq. (17). Physically, our result based on the detailed- balance-preserved QME indicates that the dot-state basis is not a proper representation to show dephasing. Under the weak measurement considered here, the qubit is weakly perturbed by the detector and its eigenstates remain a good representation to describe its dissipative dynamics. In this qubitHamiltonian dominated regime, complete dephasing is anticipated to take place between the qubit eigenstates rather than the dot states. ${ }^{7,23}$ In Fig. 3(b) we transform the offdiagonal density-matrix element $\rho_{a b}$ into $\rho_{12}$, i.e., from the dot-state basis to the eigenstate basis. A complete dephasing is observed satisfactorily between the qubit eigenstates.

So far, we have restricted our discussion in the symmetric qubit, and have already got insight in the impact of detailed balance on the qubit relaxation and dephasing. Below, we briefly show results for the asymmetric qubit, where more apparent effects can be observed.

\section{B. Asymmetric case: $\epsilon_{a} \neq \epsilon_{b}$}

In an asymmetric case, simple diagonalization of the qubit Hamiltonian gives rise to the eigenstates $|1\rangle=\cos (\theta / 2)|a\rangle$ $+\sin (\theta / 2)|b\rangle$ and $|2\rangle=\sin (\theta / 2)|a\rangle-\cos (\theta / 2)|b\rangle$; see the Appendix. We still denote the eigenenergy difference by $\Delta$ $=E_{1}-E_{2}$. With the knowledge of eigenstates, one can straightforwardly evaluate the operator $\widetilde{W}^{( \pm)}$in the master equation. In dot representation, the result reads

$$
\begin{gathered}
\widetilde{W}_{a a}^{( \pm)}=\left[T+\frac{\chi}{2}\left(1+\cos ^{2} \theta\right)\right] C^{( \pm)}(0)+\chi \lambda_{ \pm} \sin ^{2} \theta, \\
\widetilde{W}_{b b}^{( \pm)}=\left(T+\frac{\chi}{2} \sin ^{2} \theta\right) C^{( \pm)}(0)-\chi \lambda_{ \pm} \sin ^{2} \theta, \\
\widetilde{W}_{a b}^{( \pm)}=\frac{\chi}{4} C^{( \pm)}(0) \sin 2 \theta-\chi\left(\bar{\lambda}_{ \pm}+\lambda_{ \pm} \cos \theta\right) \sin \theta, \\
\widetilde{W}_{b a}^{( \pm)}=\frac{\chi}{4} C^{( \pm)}(0) \sin 2 \theta+\chi\left(\bar{\lambda}_{ \pm}-\lambda_{ \pm} \cos \theta\right) \sin \theta .
\end{gathered}
$$

In Figs. 4(a) and 4(b), the measurement-induced qubit-state relaxation is shown in (a) dot state, and (b) eigenstate representations. In the absence of detailed balance, we see in both representations that the qubit state relaxes to a statistical mixture with equal probabilities on the two states of the qu- 

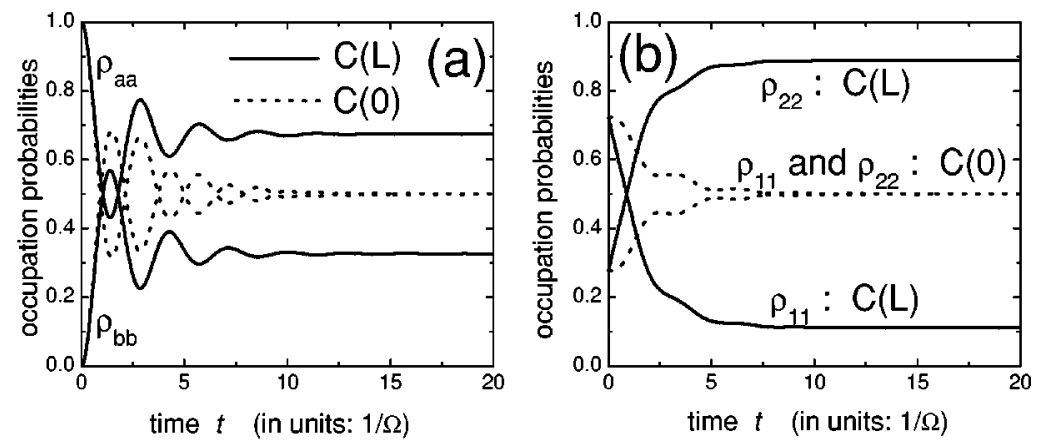

FIG. 4. Measurement-induced relaxation and dephasing of an asymmetric qubit (with dot-level offset $\epsilon_{a}-\epsilon_{b}=0.5 \Omega$ ) in, respectively, the individual dot-state representation [(a) and (c)], and the eigenstate representation [(b) and (d)]. In (a)(c), the results in the presence and the absence of
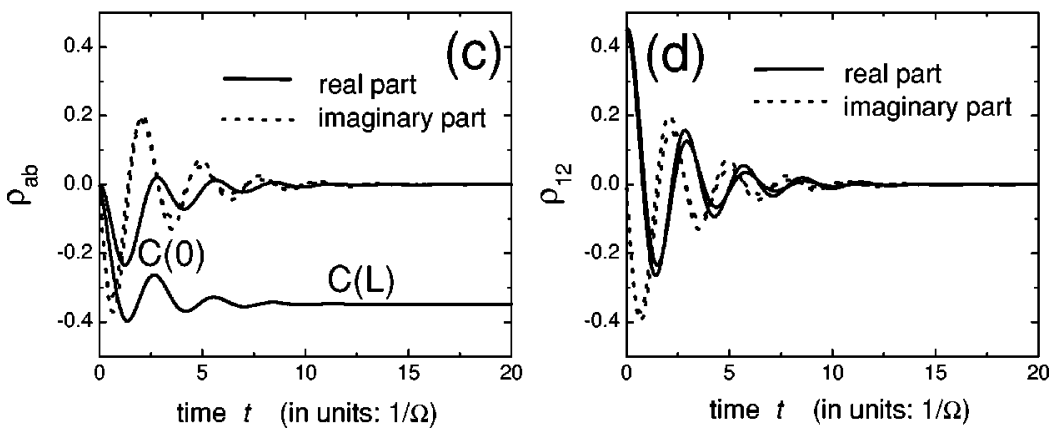
the detailed balance are symbolized by " $C(\mathcal{L})$ " and " $C(0)$." The qualitative feature of dephasing in (c) and (d) is similar to the symmetric qubit, and the corresponding figure description is referred to in Fig. 3.

bit. This peculiar feature in asymmetric qubit is owing to the equal probabilities with which the transitions from $|1\rangle$ to $|2\rangle$, and from $|2\rangle$ to $|1\rangle$, take place in the absence of detailed balance, as shown by the dominant Lindblad relaxation terms in the master equation in the Appendix. However, if the detailed balance is properly accounted for, remarkably different statistical mixture will be approached after the measurement, see the solid curves in Figs. 4(a) and 4(b). We anticipate that the relaxation behavior in Fig. 4(a) can be demonstrated by future experiment. For asymmetric qubit, the dephasing characteristics shown in Figs. 4(c) and 4(d) are similar to that in the symmetric qubit. Again, complete dephasing takes place between the eigenstates rather than the dot states of the qubit under weak measurement.

It is desirable to compare the measurement-induced relaxation described in this work (cf. Figs. 2 and 4) with that originated from coupling with an additional thermal bath as discussed in Ref. 18. In both cases, relaxations are owing to the energy exchange between the system of interest and the environment. In this sense, the relaxation induced here by a measurement device should be similar to that by a thermal bath. This analogy is also discussed in Ref. 15, where the measurement voltage across the detector is shown to be equivalent to an effective temperature of thermal bath in certain sense. Since the measurement in Ref. 18 is described as the previous work ${ }^{1}$ which causes only decoherence, an additional bath is introduced there for relaxation. It is expected that our QME approach allows the measurement itself to generate the relaxation and its consequences discussed in Ref. 18.

\section{RELAXATION AND DEPHASING RATES}

In this section we carry out the analytical expressions for relaxation and dephasing rates and discuss their characteristics which depend on the measurement conditions. Since the weak measurement under study is in the qubit-Hamiltonian dominated regime, we present our analysis in the qubiteigenstate representation (see the Appendix), in which Eq. (6) can be expressed as ${ }^{21}$

$$
\dot{\rho}_{j k}=-i \omega_{j k} \rho_{j k}-\sum_{j^{\prime}, k^{\prime}=1}^{2} \mathcal{R}_{j k, j^{\prime} k^{\prime}} \rho_{j^{\prime} k^{\prime}},
$$

where $\omega_{j k}=E_{j}-E_{k}$, and the dissipation tensor element reads

$$
\mathcal{R}_{j k, j^{\prime} k^{\prime}}=\left(\mathcal{K}_{j k, j^{\prime} k^{\prime}}+\mathcal{K}_{k j, k^{\prime} j^{\prime}}^{*}\right) / 2
$$

with

$$
\begin{aligned}
\mathcal{K}_{j k, j^{\prime} k^{\prime}}= & \delta_{k k^{\prime}}\left[W^{\dagger} \widetilde{W}^{(-)}+W \widetilde{W}^{(+) \dagger}\right]_{j j^{\prime}}-\left[W_{k k^{\prime}}^{*}, \widetilde{W}_{j j^{\prime}}^{(-)}\right. \\
& \left.+W_{k^{\prime} k} \widetilde{W}_{j^{\prime} j}^{(+) *}\right] .
\end{aligned}
$$

These tensor elements have clear physical meaning. For example, $-\mathcal{R}_{j j, k k}$ (with $j \neq k$ ) amounts to transfer of the occupation probability from $|k\rangle$ to $|j\rangle$, while $\mathcal{R}_{j k, j k}$ describes the dephasing between $|j\rangle$ and $|k\rangle$. This can be further elucidated by making the so-called secular approximation which retains only the diagonal relaxation tensor elements, such as $\mathcal{R}_{j j, k k}$ and $\mathcal{R}_{j k, j k}$. In the qubit-eigenstate basis $\{|1\rangle,|2\rangle\}$, the secular approximation leads to the following Bloch equation:

$$
\begin{gathered}
\dot{\rho}_{11}=-\dot{\rho}_{22}=-\Gamma_{1} \rho_{11}+\Gamma_{2} \rho_{22}, \\
\dot{\rho}_{12}=-i \Delta \rho_{12}-\gamma_{12} \rho_{12},
\end{gathered}
$$

where

$$
\begin{gathered}
\Gamma_{1} \equiv-\mathcal{R}_{22,11}=\left|W_{12}\right|^{2}\left[C^{(-)}(\Delta)+C^{(+)}(\Delta)\right], \\
\Gamma_{2} \equiv-\mathcal{R}_{11,22}=\left|W_{21}\right|^{2}\left[C^{(-)}(-\Delta)+C^{(+)}(-\Delta)\right],
\end{gathered}
$$




$$
\begin{aligned}
\gamma_{12} \equiv \mathcal{R}_{12,12}= & \frac{1}{2}\left(\Gamma_{1}+\Gamma_{2}\right)+\frac{1}{2}\left(W_{11}-W_{22}\right)^{2}\left[C^{(-)}(0)\right. \\
& \left.+C^{(+)}(0)\right]
\end{aligned}
$$

The relaxation between $|1\rangle$ and $|2\rangle$ is characterized by the evolution of $\rho_{z}(t) \equiv \rho_{11}(t)-\rho_{22}(t)$. From Eq. (22a) it is easy to show that

$$
\dot{\rho}_{z}(t)=-\left(\Gamma_{1}+\Gamma_{2}\right)\left[\rho_{z}(t)-\rho_{z}(\infty)\right],
$$

which results in the solution

$$
\rho_{z}(t)=\rho_{z}(\infty)+\left[\rho_{z}(0)-\rho_{z}(\infty)\right] e^{-\left(\Gamma_{1}+\Gamma_{2}\right) t} .
$$

Accordingly, the $T_{1}$-relaxation rate is obtained as

$$
\frac{1}{T_{1}}=\Gamma_{1}+\Gamma_{2}=\frac{g_{\mathrm{L}} g_{\mathrm{R}}}{2 / \pi}[F(e V+\Delta)+F(e V-\Delta)] \chi^{2} \sin ^{2} \theta,
$$

with

$$
F(x) \equiv x \operatorname{coth}(\beta x / 2) .
$$

Similarly, the $T_{2}$-dephasing rate can be obtained as [cf. Eq. (22b)]

$$
\frac{1}{T_{2}}=\gamma_{12}=\frac{1}{2 T_{1}}+\pi g_{\mathrm{L}} g_{\mathrm{R}} F(e V) \chi^{2} \cos ^{2} \theta .
$$

In this result, the first term stems from the relaxation-induced dephasing and the second term describes the pure dephasing. This identification can be simply understood as follows. In the eigenstate representation (see the Appendix), the qubit and interaction Hamiltonians read, respectively, $H_{\text {qu }}$ $=(\Delta / 2) \sigma_{z} \quad$ and $\quad H^{\prime}=W X=\left[(T+\chi) I+(\chi / 2)\left(\cos \theta \sigma_{z}\right.\right.$ $\left.\left.+\sin \theta \sigma_{x}\right)\right] X$. From a master equation based analysis, ${ }^{22}$ one can easily prove that the $\sigma_{x}$ coupling would cause the $T_{1}$ relaxation with rate $1 / T_{1}$ given by Eq. (26), and simultaneously induce dephasing with rate $1 /\left(2 T_{1}\right)$. Meanwhile, the $\sigma_{z}$ coupling only results in pure dephasing with rate given by the second term of Eq. (28).

Equations (26) and (28) describe the dependence of the qubit relaxation and dephasing rates on the various measurement parameters. Most apparently, the rates depend on the visibility parameter $\chi$ via $\propto \chi^{2}$, which is the result in weakcoupling regime, but implies also that design of an appropriately large $\chi$ is essential in order to perform efficient measurement. The dependence of the relaxation and dephasing rates on the measurement voltage and temperature is numerically plotted in Fig. 5. In general, both the applied voltage and temperature will enhance the qubit relaxation and dephasing. Dephasing will, in principle, benefit quantum measurement. However, in practice the detector should be kept at very low temperatures, since there exists a tradeoff between the signal and noise strengths in the detector, analysis on which has appeared in recent publications. ${ }^{6,9}$ In what follows, based on Eqs. (26) and (28), we detail the voltage and temperature dependence of the relaxation and dephasing rates under certain limits. First, in the limit of zero-bias voltage across the detector, the temperature dependence of the relaxation rate is characterized by $T_{1}^{-1} \propto \operatorname{coth}\left(\Delta / 2 k_{B} T\right)$,

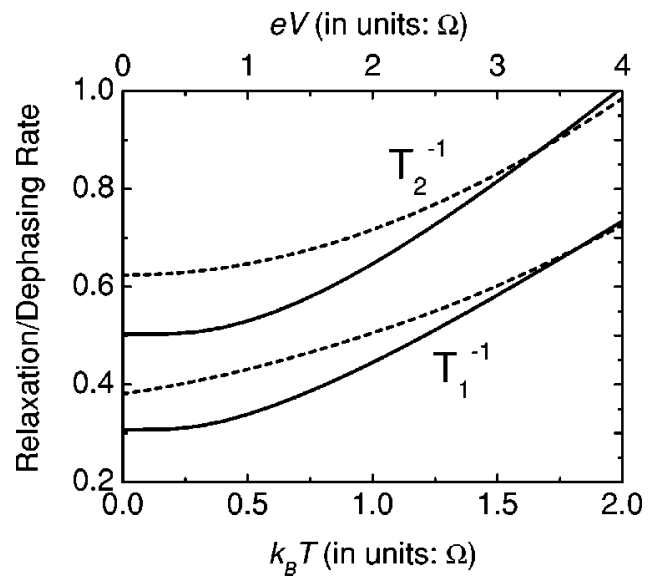

FIG. 5. The measurement-induced qubit relaxation rate $\left(T_{1}^{-1}\right)$ and dephasing rate $\left(T_{2}^{-1}\right)$ as functions of the temperature for fixed voltage $e V=\Omega$ (solid curves), and of the measurement voltage for fixed temperature $k_{B} T=\Omega$ (dashed curves).

whereas the $\sigma_{z}$-coupling induced pure-dephasing rate, i.e., the second term in Eq. (28), is $\propto k_{B} T$. This difference in temperature dependence is due to that energy exchange between the qubit and detector takes place during relaxation, but there exists no such exchange during pure dephasing. Under zero-bias voltage, the detector no longer plays the role of measurement, the qubit relaxation and dephasing are merely caused by the zero-voltage quantum and thermal fluctuations due to random tunneling of electrons through the QPC. Second, at zero-temperature limit, the pure-dephasing rate linearly depends on the measurement voltage by noting that $F(e V) \propto e V$. Interestingly, the relaxation rate reduces to $T_{1}^{-1} \propto(e V+\Delta+|e V-\Delta|)$, which linearly depends on the voltage if $e V>\Delta$, but becomes a voltage-independent constant when $e V<\Delta$.

\section{CONCLUSION}

In summary, we have studied the relaxation and dephasing of a solid-state charge qubit under quantum measurement of a mesoscopic detector. Our treatment emphasizes in particular the energy exchange between the qubit and detector during the measurement process. The measurement current fluctuation is shown to have significant impact on not only the decoherence but also the detailed-balance-preserved relaxation of qubit. We have carried out both numerical and analytical results for the qubit relaxation and dephasing, and highlighted the important features which might be relevant to future experiments. Our unified QME approach is expected to be generalized to a conditional version which enables to study the readout statistics, and to be unraveled by stochastic wave function which can describe an individual continuous measurement of a single qubit. The work along these two lines is in progress and will be published elsewhere.

\section{APPENDIX: ELABORATION ON THE DETAILED BALANCE}

In this appendix we carry out the explicit operator form for the relaxation superoperator $R \rho$, from which the detailed 
balance retained by our QME can be revealed clearly. To this end, we express the qubit operators in the eigenstate representation. In general, for asymmetric qubit, the individual dot level $\epsilon_{a} \neq \epsilon_{b}$, we introduce $\epsilon=\left(\epsilon_{a}-\epsilon_{b}\right) / 2$ for the dot-level offset, and $\Delta=E_{1}-E_{2}$ for the qubit eigenenergy difference. By a simple diagonalization of the qubit Hamiltonian, the eigenenergies are obtained as $E_{1}=\sqrt{\epsilon^{2}+\Omega^{2}}$ and $E_{2}$ $=-\sqrt{\epsilon^{2}+\Omega^{2}}$. Correspondingly, the eigenstates are $|1\rangle$ $=\cos (\theta / 2)|a\rangle+\sin (\theta / 2)|b\rangle \quad$ and $\quad|2\rangle=\sin (\theta / 2)|a\rangle$ $-\cos (\theta / 2)|b\rangle$, where $\theta$ is introduced by $\cos \theta=\epsilon / \sqrt{\epsilon^{2}+\Omega^{2}}$, and $\sin \theta=\Omega / \sqrt{\epsilon^{2}+\Omega^{2}}$. In the eigenstate basis $\{|1\rangle,|2\rangle\}$, the qubit Hamiltonian reads $H_{\mathrm{qu}}=(\Delta / 2) \sigma_{z}$, and the coupling between the qubit and detector is described by $H^{\prime}=W X$ $=\left[(T+\chi) I+(\chi / 2)\left(\cos \theta \sigma_{z}+\sin \theta \sigma_{x}\right)\right] X$. Here $I$ is the $2 \times 2$ unit matrix, $X$ stands for the tunneling operator of the QPC, and the Pauli operators $\sigma_{z}=|1\rangle\langle 1|-| 2\rangle\langle 2|$ and $\sigma_{x}$ $=|1\rangle\langle 2|+| 2\rangle\langle 1|$, which map the two-state qubit to a spin$1 / 2$ particle.

In terms of the Pauli matrices, the formal QME, Eq. (5), can be recast to an explicit form with

$$
\begin{aligned}
\mathcal{R} \rho= & \eta_{1}^{2} C(0)\left[\sigma_{z},\left[\sigma_{z}, \rho\right]\right]+\eta_{2}^{2}\left[\sigma_{x}, \widetilde{Q}_{x} \rho-\rho \widetilde{Q}_{x}^{\dagger}\right] \\
& +\eta_{1} \eta_{2} C(0)\left[\sigma_{x},\left[\sigma_{z}, \rho\right]\right]+\eta_{1} \eta_{2}\left[\sigma_{z}, \widetilde{Q}_{x} \rho-\rho \widetilde{Q}_{x}^{\dagger}\right],
\end{aligned}
$$

where $\quad \eta_{1}=(\chi / 2) \cos \theta, \quad \eta_{2}=(\chi / 2) \sin \theta, \quad C(0)=C^{(+)}(0)$ $+C^{(-)}(0)$, and $\widetilde{Q}_{x} \equiv C(-\mathcal{L}) \sigma_{x}$ with $C(-\mathcal{L}) \equiv C^{(+)}(-\mathcal{L})$ $+C^{(-)}(-\mathcal{L})$. In the right-hand side of Eq. (A1), the first term describes the $\sigma_{z}$-coupling induced pure dephasing, the second term dominantly contributes the $T_{1}$ relaxation as well as its associated dephasing owing to the $\sigma_{x}$ coupling, and the last two terms stem from the correlation between the two couplings which have minor contribution to the dissipative dynamics. Due to the dominant $T_{1}$-rate contribution of the second term, we further express it into a Lindblad-type form,

$$
\begin{aligned}
{\left[\sigma_{x}, \widetilde{Q}_{x} \rho-\rho \widetilde{Q}_{x}^{\dagger}\right]=} & -2 C(-\Delta) \mathcal{D}\left[\sigma^{+}\right] \rho-2 C(\Delta) \mathcal{D}\left[\sigma^{-}\right] \rho \\
& -[C(-\Delta)+C(\Delta)]\left(\sigma^{-} \rho \sigma^{-}+\sigma^{+} \rho \sigma^{+}\right),
\end{aligned}
$$

where the Lindblad superoperators are defined as

$$
\mathcal{D}\left[\sigma^{+}\right] \rho=\sigma^{+} \rho \sigma^{-}-\frac{1}{2}\left\{\sigma^{-} \sigma^{+}, \rho\right\},
$$

$$
\mathcal{D}\left[{\sigma^{-}}^{-} \rho=\sigma^{-} \rho \sigma^{+}-\frac{1}{2}\left\{\sigma^{+} \sigma^{-}, \rho\right\} .\right.
$$

In deriving this result, we have carried out $\widetilde{Q}_{x}=C(-\Delta) \sigma^{+}$ $+C(\Delta) \sigma^{-}$, where $\sigma^{ \pm}=\frac{1}{2}\left(\sigma_{x} \pm i \sigma_{y}\right)$. In this context, simple algebras were used as follows. Note that $\mathcal{L} \sigma_{x} \equiv\left[H_{\mathrm{qu}}, \sigma_{x}\right]$ $=(\Delta / 2)(2 i) \sigma_{y}, \mathcal{L}^{2} \sigma_{x}=(\Delta / 2)^{2}(2 i)(-2 i) \sigma_{x}$, and so on. It then follows that the action of an arbitrary function of the Liouvillian operator $\mathcal{L}$, say, $C(\mathcal{L})$, on $\sigma_{x}$ reads $C(\mathcal{L}) \sigma_{x}$ $=C_{1}(\Delta) \sigma_{x}+i C_{2}(\Delta) \sigma_{y}$, with $C_{1}(\Delta)=[C(\Delta)+C(-\Delta)] / 2$ and $C_{2}(\Delta)=[C(\Delta)-C(-\Delta)] / 2$.

The terms " $\sigma^{-} \rho \sigma^{-}$" and " $\sigma^{+} \rho \sigma^{+}$" in Eq. (A2) are out of the rotating-wave approximation and their effects are small compared with the Lindblad terms with $\mathcal{D}\left[\sigma^{ \pm}\right] \rho$ [Eq. (A3)]. Physically, $\mathcal{D}\left[\sigma^{-}\right] \rho$ describes quantum jump from the upper qubit state $|1\rangle$ to the lower state $|2\rangle$, and $\mathcal{D}\left[\sigma^{+}\right] \rho$ vice versa. With satisfaction, the corresponding jump probability $C( \pm \Delta)$ precisely relates the qubit jump to the electron tunneling in the detector in the presence of energy-quanta (i.e., $\Delta$ ) emission (absorption). Note that this energy exchange is essential to ensure the detailed-balance. Denoting the occupation probabilities on the qubit states $|1\rangle$ and $|2\rangle$ by $P_{1}$ and $P_{2}$, at the stationary mixture state, the dominant term of Eq. (A2) leads to $P_{1} / P_{2}=C(-\Delta) / C(\Delta)$. This is nothing but a generalization of the usual detailed balance relation for coupling with a thermal bath. Here the measurement voltage plays certain role of an effective temperature. This result is also in complete consistence with the rate analysis in Sec. IV, see Eqs. (22) and (23). If we neglect the energy exchange, say, let $C( \pm \Delta) \rightarrow C(0)$, the detailed balance is broken down, and equal occupation probabilities on the qubit states are inevitably obtained as in the previous literatures. ${ }^{1,5}$

Note added in proof. Recently, we were informed by T.M. Stace that in their recent work, ${ }^{24}$ the energy exchange between the qubit and detector (i.e., the inelastic tunneling in the detector) has also been considered.

\section{ACKNOWLEDGMENTS}

Support from the Major State Basic Research Project No. G001CB3095 of China, the Special Fund for "100 Person Project" from Chinese Academy of Sciences, the National Natural Science Foundation of China, and the Research Grants Council of the Hong Kong Government is gratefully acknowledged.
${ }^{1}$ S.A. Gurvitz, Phys. Rev. B 56, 15215 (1997).

${ }^{2}$ I.L. Aleiner, N.S. Wingreen, and Y. Meir, Phys. Rev. Lett. 79, 3740 (1997).

${ }^{3}$ Y. Levinson, Europhys. Lett. 39, 299 (1997); Phys. Rev. B 61, 4748 (2000).

${ }^{4}$ L. Stodolsky, Phys. Lett. B 459, 193 (1999).

${ }^{5}$ H.S. Goan, G.J. Milburn, H.M. Wiseman, and H.B. Sun, Phys. Rev. B 63, 125326 (2001).

${ }^{6}$ S. Pilgram and M. Büttiker, Phys. Rev. Lett. 89, 200401 (2002).

${ }^{7}$ A. Shnirman and G. Schön, Phys. Rev. B 57, 15400 (1998); Y.
Makhlin, G. Schön, and A. Shnirman, Phys. Rev. Lett. 85, 4578 (2000); Rev. Mod. Phys. 73, 357 (2001).

${ }^{8}$ A.N. Korotkov, Phys. Rev. B 60, 5737 (1999); 63, 085312 (2001).

${ }^{9}$ D.V. Averin, cond-mat/0004364 (unpublished); A.N. Korotkov and D.V. Averin, cond-mat/0002203 (unpublished).

${ }^{10}$ A.A. Clerk, S.M. Girvin, A.K. Nguyen, and A.D. Stone, Phys. Rev. Lett. 89, 176804 (2002).

${ }^{11}$ M.H. Devroret and R.J. Schoelkopf, Nature (London) 406, 1039 (2000).

${ }^{12}$ E. Buks, R. Schuster, M. Heiblum, D. Mahalu, and V. Umansky, 
Nature (London) 391, 871 (1998); D. Sprinzak, E. Buks, M. Heiblum, and H. Shtrikman, Phys. Rev. Lett. 84, 5820 (2000).

${ }^{13}$ Y. Nakamura, Yu.A. Pashkin, and J.S. Tsai, Nature (London) 398, 786 (1999).

${ }^{14}$ S.A. Gurvitz, Phys. Rev. Lett. 85, 812 (2000).

${ }^{15}$ D. Mozyrsky and I. Martin, Phys. Rev. Lett. 89, 018301 (2002).

${ }^{16}$ A. Schnirman, D. Mozyrsky, and I. Martin, cond-mat/0211618 (unpublished).

${ }^{17}$ R.J. Schoelkopf, A.A. Clerk, S.M. Girvin, K.W. Lehnert, and M.H. Devoret, cond-mat/0210247 (unpublished).

${ }^{18}$ S.A. Gurvitz, L. Fedichkin, D. Mozyrsky, and G.P. Berman, Phys. Rev. Lett. 91, 066801 (2003).

${ }^{19}$ Xin-Qi Li and Y. Arakawa, Phys. Rev. A 63, 012302 (2001).
${ }^{20}$ Y.J. Yan, Phys. Rev. A 58, 2721 (1998).

${ }^{21}$ R.X. Xu, Y.J. Yan, and X.Q. Li, Phys. Rev. A 65, 023807 (2002).

${ }^{22}$ Y.J. Yan, F. Shuang, R.X. Xu, J.X. Cheng, X.Q. Li, Y. Yang, and H.Y. Zhang, J. Chem. Phys. 113, 2068 (2000).

${ }^{23}$ In the regime of strong (projective) measurement, the qubitdetector interaction Hamiltonian will dominate the qubit dynamics. Accordingly, complete dephasing between the individual dot states (i.e., the eigenstates of the observable) is anticipated. However, the usual perturbative-theory-based QME approach does not work in this regime.

${ }^{24}$ T.M. Stace and S.D. Barrett, cond-mat/0307727 (unpublished); cond-mat/0309610 (unpublished). 